\title{
Percutaneous Full Endoscopic Transverse Processectomy for Bertolotti's Syndrome
}

\author{
Jong Duck Yoo, Pius Kim, Chang Il Ju, Seung Myung Lee \\ Department of Neurosurgery, Chosun University College of Medicine, Gwangju, Korea
}

Corresponding Author: Chang Il Ju, MD, PhD Department of Neurosurgery, Chosun University College of Medicine, 365 Pilmun-daero, Dong-gu, Gwangju 61453, Korea

Tel: $+82-62-220-3126$ Fax: +82-62-227-4575

E-mail: jchangil@hanmail.net

Received: May 12, 2019 Revised: May 30, 2019 Accepted: June 5, 2019

The Lumbosacral transitional vertebra (LSTV) is a congenital anomaly and has two types of abnormal vertebrae. Low back pain and leg pain originated from a lumbosacral transitional vertebra (LSTV) known as Bertolotti's syndrome (BS). We describe our institution's experience of a L5 transverse processectomy done via a percutaneous endoscopic approach for a patient with Bertolotti's syndrome. A 64year-old female patient with persistent left leg radiating pain visited at the spine center. This patients underwent endoscopic L5-S1 foraminoplasty under the diagnosis of foraminal stenosis. However, despite the complete neural decompression, the patient complained of persistent left leg pain. We found that the left transverse process (TP) of L5 vertebra seemed to contact with the ala of sacrum suggesting the Bertolotti's syndrome (BS). A pseudo-articulation lidocaine injection was given and it was effective in reducing the leg pain. Under local anesthesia, a uniportal endoscopy was introduced to the base of the L5 TP and simply cut in cranial to caudal direction using a high-speed drill in order to block the way of mechanical stress from spine. The patient's symptoms got relieved after $L 5$ transverse processectomy and she was discharged in a few days.

Key Words: Lumbosacral transitional vertebra (LSTV), Percutaneous full endoscopic surgery, Transverse process

\section{INTRODUCTION}

Low back pain and leg pain originated from a lumbosacral transitional vertebra (LSTV) known as Bertolotti's syndrome (BS). It was first described by Mario Bertolotti in 1917 and it features an increase in size of unilateral or bilateral transverse process (TP) of the most caudal lumbar spine. In addition, it also forms pseudo-articulation or bony fusion between enlarged TP and the ala of sacrum ${ }^{1)}$.

While BS is not recognized enough in clinical practice by plenty of physicians, some reports suggest that it is not known to be a rare condition. There was a study that $35.6 \%$ as positive for a LSTV in 211 radiographs ${ }^{2}$. Moreover, it was reported that neural compression by LSTV was demonstrated in $13 \%$ of 74 patients whose transitional vertebrae were found in the report of MRI scan ${ }^{3)}$. Despite the prevalence rate like those as several reports have shown, symptomatic patients who have BS often receive inadequate treatment, owing to misdiagnosis at an early stage $^{4,5)}$.

Current operative techniques for treating BS are normally based on the procedure including decompression of L5 nerve root as well as resection of the pseudo-articulations or the part of TP which contact with ala of sacrum. In recent years, minimally invasive techniques have been reported by a large number of authors ${ }^{6-9)}$. However, there are still only a few reports on percutaneous full endoscopic surgery for BS.

In recent years, endoscopic techniques for spinal surgery have been surprisingly developing and gradually replacing conventional surgical techniques. In line with this trend, we tried to perform a full endoscopy for treating BS and finally we have achieved a successful outcome in a case. This report offers a detailed description about the operative procedure and technical differences with full percutaneous endoscopy compared to other previous skills.

\section{CASE DESCRIPTION}

A 64-year-old woman with a few years history of left leg pain which got severely worsened a week ago visited at the spine center. In a neurologic examination, there was no abnormal sign such as decreased anal tone, muscle weakness or sensory change. The patient had already received numeral spine surgeries several years ago - decompressive ligamentectomy at L3-L4 and posterior lumbar interbody fusion at L4-L5 for spinal stenosis eight years earlier. In spite of those surgeries, she suffered from remained low back pain and radiating pain over the years. For that reason, she had received countless times of medial 
bundle branch block or facet block at lumbar spine. Furthermore, two years earlier from the visit, she had severe right leg pain and sought intra-discal electrothermal therapy for foraminal disc herniation at the right side of L5-S1. After she was examined by lumbar MRI scan in our institute, foraminal stenosis either L3-4 or L5-S1 at the left side (Fig. 1) was discovered. Because the stenosis at the L5-S1 seemed to be dominant rather than the one at the L3-4, a diagnostic lidocaine injection was selectively provided on the left $\mathrm{L} 5$ nerve root. Then the patient experienced temporary relief of leg pain. Thus, we were able to make a diagnosis that the left leg pain had been originated
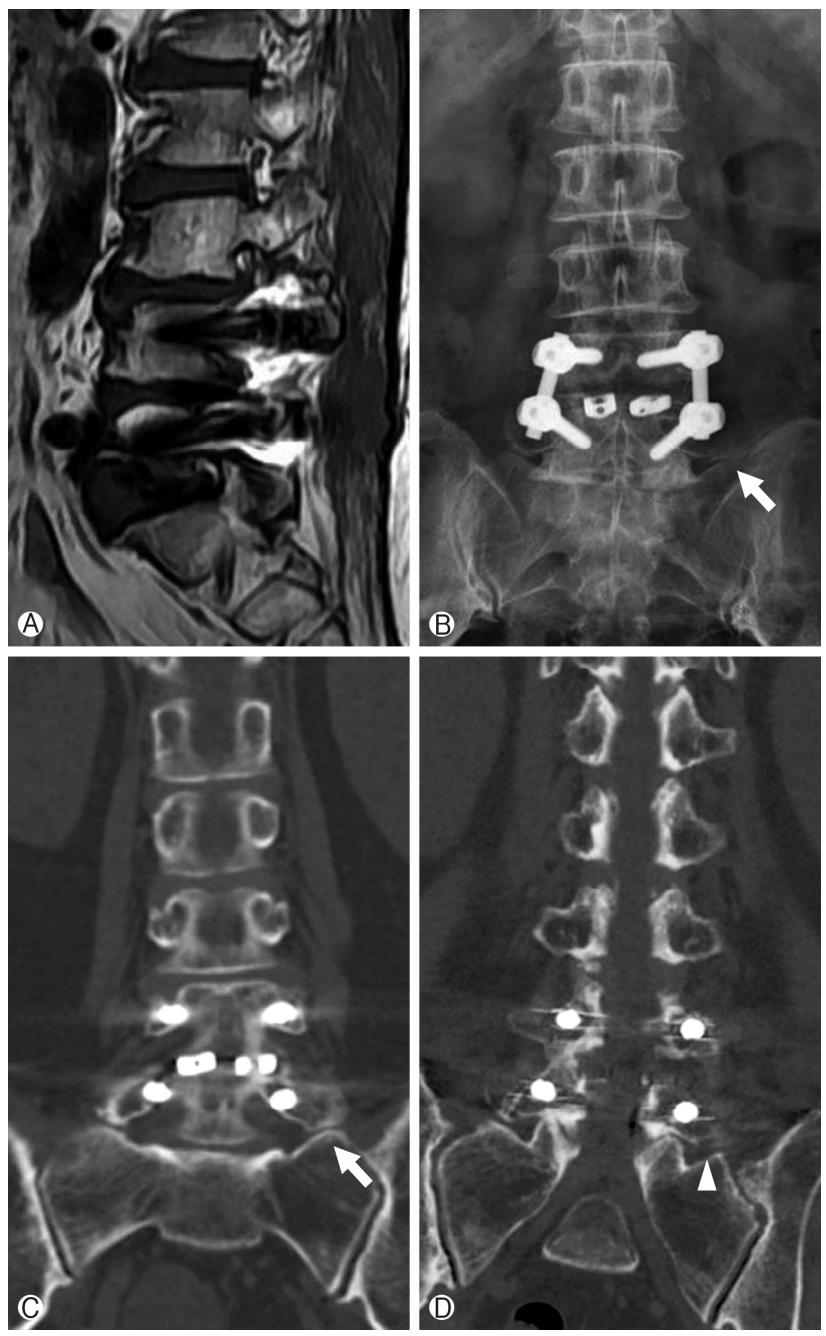

Fig. 1. (A) Lumbar MRI demonstrated left foraminal stenosis above and below the level of previous interbody fusion due to subsequent adjacent segment degeneration. The bottom one showed more severe stenosis. (B) A bone to bone contact between TP of L5 vertebra and ala of sacrum was seen at the left side on simple anteroposterior radiograph (white arrow). (C) Preoperative lumbar CT scan showed an explicit evidence of bony contact (white arrow). (D) Postoperative lumbar CT scan showed a complete cutting of base of the left TP of L5 in cranial to caudal direction (white arrow head). from the left foraminal stenosis of $\mathrm{L} 5-\mathrm{S} 1$ causing $\mathrm{L} 5$ radiculopathy. Before long the patient had a foraminotomy at the left foramen of L5-S1 by percutaneous full endoscopy using a uniportal spine endoscopic device. In that operation, the herniated disc on the foraminal side was resected by forcep along with intradiscal nucleus pulposus more inside, the ligaments which may compress nerve root were totally removed and the ipsilateral superior articular process was partially drilled out. Through all these procedures without any complications, we made sure that the left $L 5$ nerve root had been totally decompressed by a skilled technique. However, despite the complete neural decompression, the patient still felt sustained leg pain. She complained that her leg pain had not improved at all. Soon, all the radiologic images were fully reviewed again to find out any other missed lesion since our initial diagnosis might have been wrong. Finally, on anteroposterior view from a simple radiograph of lumbar spine, we identified that the left TP of L5 vertebra seemed to contact with the ala of sacrum suggesting the BS. Thus we performed a pseudo-articularis lidocaine injection so as to exclude it. As a result, it turned out to be strongly effective in reducing leg pain. Then, we were able to make a definite diagnosis with BS for this patient. In a few days, she underwent a transverse processectomy by percutaneous full endoscopy using a uniportal spine endoscopic device. By a posterolateral approach, the left TP of L5 was simply cut at the base part of it. Consequently, any complications were not found during the surgical procedure. At last, the patient's symptoms got relieved right after the surgery and she recovered in a few days.

\section{OPERATIVE TECHNIQUE}

Firstly, under local anesthesia and C-arm visualization, the needle and guidewire were placed over the L5 TP. In the same manner, as a percutaneous endoscopic transforaminal lumbar discectomy, an obturator, a cannula, and a uniportal endoscopy were sequentially introduced to the L5 TP. By an endoscopic operative view, cranial and caudal margin at the base part of the TP were clearly identified. Secondly, the base of the L5 TP was simply cut in cranial to caudal direction using a high-speed drill. Thirdly, connective tissue and ligament on the anterior side of the TP were visualized which were dissected and removed by a forcep and a Kerrison punch. Upon that the $L 4$ nerve root passing through the ventral side of TP and surrounded by fat tissue was visualized and we confirmed that it was free (Fig. 2). Moving the view of the endoscope to the caudal side, the L5nerve root traversing caudal to the TP was also identified and confirmed that it was free. After checking thoroughly, in the field of the endoscopic vision whether the TP of L5 was completely resected and the $L 4$ and $L 5$ nerve roots were free from neural compression, we finished the operation

\section{DISCUSSION}

LSTV is a congenital anomaly and has two types of abnormal vertebrae. The first type is that the L 5 vertebra is fused with 

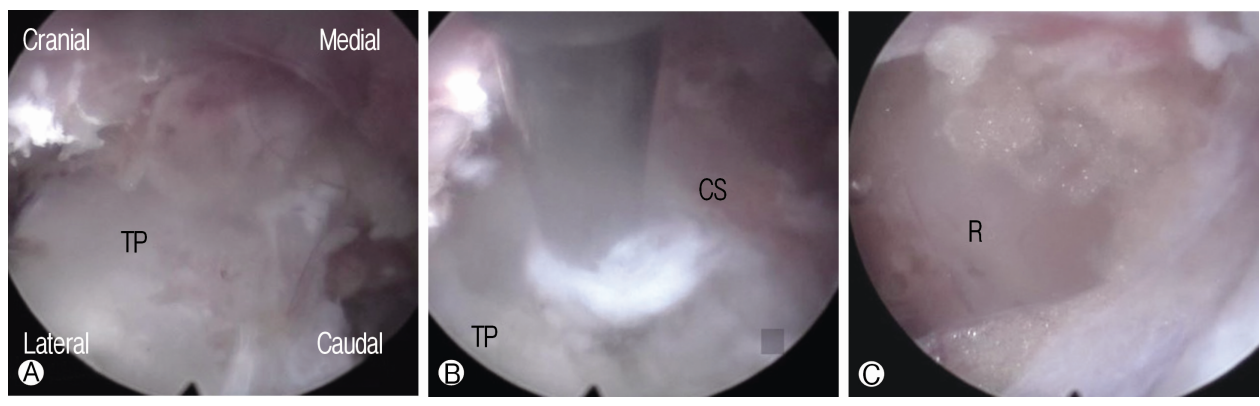

Fig. 2. A Intraoperative endoscopic view after full exposure of transverse process. (B) A complete cutting was done in cranial to caudal direction by high-speed drill, and then the cut surface (CS) of base of TP was observed. (C) $L 4$ nerve root(R) passing in just front of TP was visualized after decompressive processectomy.

the S1 vertebra which called "sacralization of L5". And the other one is that the S1 vertebra is separated from S2 without fusion which is called "lumbarization of S1"10,11). The features of the correlation between the TP of LSTV and the uppermost sacral bone may vary. They were classified into four types morphologically by Castellvi et al. ${ }^{12)}$. According to whether they form a pseudo-articulation or bony fusion. Where the pain comes from in BS depends on how the LSTV and the sacrum forma intersegmental relationship according to those morphological types.

The mechanism that BS causes pain is diverse. Some studies indicated that it was caused by the degeneration of pseudo-articulation between a LSTV and an ala of sacrum ${ }^{13-15)}$. It was also proved that the pain came from $L 5$ nerve root entrapment by extraforaminal stenosis on account of enlarged TP of LSTV, called far out syndrome ${ }^{16-19)}$. Disc degeneration above LSTV subsequent to BS was also reported as a possible cause of pain $^{11,20)}$. In addition, it was suggested that facet joint on the opposite side of the fused pseudo-articularis could cause pain $^{21,22)}$. As such, the pain of BS might occur in various lesions around the TP of LSTV and ala of sacrum. Therefore a definite differentiation to clarify whether the pain originated from has to precede surgery.

There are variable surgical techniques for symptomatic BS which is untreatable by conservative treatment. They consist of two main methods such as an intersegmental fusion between LSTV, sacrum and a resection of the pseudo-articulation. Santavirta et al. ${ }^{23)}$ reported clinical outcomes of patients who underwent posterolateral fusion for symptomatic BS. Their clinical results were only a moderate level, and problems were indicated about the second operation and adjacent segment degeneration. And the authors pointed out that the results with posterolateral fusion were only slightly better than those with conservative treatment in a comparable group. Whereas, many authors reported favorable results of technique to simply resect the pseudo-articulation or decompress entrapment of the $L 5$ nerve $\operatorname{root}^{6-8,13,24-26)}$. Some of them reported minimal invasive technique using a tubular retractor and suggested that it was recommended as an effective procedure. Additionally, Ko Ikuta et al. ${ }^{6}$ applied a spinal endoscopy through the tubular retractor, and reported that it provided a well-illuminated operative field and surgical safety. It is true that those techniques were less invasive than pre-existing other conventional techniques. In fact, they are not able to be recognized as a true minimally invasive surgery. The first report introduced a percutaneous full endoscopic surgery for BS was given by Paudel et al. ${ }^{27)}$ in 2017. A uniportal spine endoscopic device was used without a tubular retractor to resect the pseudo-articulation and decompress the nerve root with a high-speed drill by direct dorsal or posterolateral approach. Then, they proposed this technique as an alternative treatment method in cases of symptomatic BS, as it preserves the iliolumbar ligament and provides early postoperative recovery compared to other existing techniques.

Meanwhile, in our institute, Ju et al. ${ }^{28)}$ reported somewhat unique surgical technique for BS in 2017. With a $2 \mathrm{~cm}$ skin incision and through the anatomic paraspinal intermuscular approach, the basal part of L5 TP and the ala of sacrum was exposed. Using a microscope, just cutting the base of TP was performed with a high-speed drill in cranial to caudal direction. This technique provides a permanent blocking the path of mechanical stress from the vertebrae to the pseudo-articulation via the TP, then it makes the pain in the pseudo-articulation get improved. This simple procedure has a few more advantages. Since articular surface of the pseudo-articulation which has to be resected by surgery usually has a relatively more irregular structure, just cutting the base of TP is much easier than the direct resection of pseudo-articulation. In addition, this method facilitates decompression of nerve roots. Just below the extension line of the TP incision line, the L5 nerve root can be identified and decompressed without difficulty. In particular, the author emphasized that $L 4$ nerve root, as well as L5 nerve root, could be affected unexpectedly by the enlarged TP of LSTV. After cutting the base of TP and dissecting the connective tissue, then the $L 4$ nerve root crossing down the ventral side of TP is able to be visualized and decompressed right away.

In this study, we attempted to implement the technique which is described above as a percutaneous full endoscopy on a patient diagnosed with BS. After the surgery, the patient was satisfied with the pain relief that had suffered over the years and no longer complained of low back pain and leg pain. Moreover, 
there were no postoperative complications. Consequently, our innovative technique worked successfully. In comparison with conventional methods, the percutaneous full endoscopic transverse proccessectomy provides that a few advantages-much less invasiveness, shorter operating time, less bleeding, and feasibility under local anesthesia. Overall, it was fully able to be performed almost the same procedure that was done by classical approaches.

\section{CONCLUSION}

$\mathrm{BS}$ is a diagnosis of exclusion. All patients who have $\mathrm{L} 4$ radiculopathy as well as L 5 radiculopathy or axial pain should be doubtful about having BS. The pain origins around the LSTV and sacrum have to be precisely differentiated before surgery. Percutaneous full endoscopic surgery is feasible, effective and safe for BS. In conclusion, we expect that our findings could probably replace the other current surgical methods.

\section{REFERENCES}

1. Bertolotti, M: Contribution to the knowledge of the defects as it relates to regional differences in the spine with special regards to the assimilation of L5 and the sacrum. Radiologique Medica (Torino) 4:113-144, 1917

2. Apazidis A, Ricart PA, Diefenbach CM, Spivak JM: The prevalence of transitional vertebrae in the lumbar spine. The Spine Journal 11(9):858-862, 2011

3. Porter NA, Lalam RK, Tins BJ, Tyrrell PN, Singh J, CassarPullicino VN: Prevalence of extraforaminal nerve root compression below lumbosacral transitional vertebrae. Skeletal Radiol 43(1):55-60, 2014

4. Manmohan S, Dzulkarnain A, Nor Azlin ZA, Fazir M: Bertolotti's syndrome: A commonly missed cause of back pain in young patients. Malays Fam Physician 10(2):55-58, 2015

5. Kundi M, Habib M, Babar S, Kundi AK, Assad S, Sheikh A: Transitional vertebra and spina bifida occulta related with chronic low back pain in a young patient. Cureus 8(10):837, 2016

6. Ikuta K, Kitamura T, Masuda K, Hotta K, Senba H, Shidahara S: Minimally invasive transtubular endoscopic decompression for L5 radiculopathy induced by lumbosacral extraforaminal lesions. Asian Spine J 12(2):246-255, 2018

7. Li Y, Lubelski D, Abdullah KG, Mroz TE, Steinmetz MP: Minimally invasive tubular resection of the anomalous transverse process in patients with Bertolotti's syndrome: presented at the 2013 Joint Spine Section Meeting: clinical article. J Neurosurg Spine 20(3):283-290, 2014

8. Takata Y, Sakai T, Higashino K, Goda Y, Mineta K, Sugiura K, et al: Minimally invasive microendoscopic resection of the transverse process for treatment of low back pain with bertolotti's syndrome. Case Rep Orthop 2014:613971, 2014

9. Yousif S, Wood M: Minimally invasive resection of lumbosacral pseudojoint resulting in complete resolution of a lower back pain - A case report and review of Bertolotti syndrome. J Clin Neurosci 51:67-68, 2018

10. Hughes RJ, Saifuddin A: Imaging of lumbosacral transitional vertebrae. Clin Radiol 59(11):984-991, 2004

11. Luoma K, Vehmas T, Raininko R, Luukkonen R, Riihimäki H:
Lumbosacral transitional vertebra: relation to disc degeneration and low back pain. Spine (Phila Pa 1976) 29(2):200-205, 2004

12. Castellvi AE, Goldstein LA, Chan DP: Lumbosacral transitional vertebrae and their relationship with lumbar extradural defects. Spine (Phila Pa 1976) 9(5):493-495, 1984

13. Malham GM, Limb RJ, Claydon MH, Brazenor GA: Anterior pseudoarthrectomy for symptomatic Bertolotti's syndrome. J Clin Neurosci 20(12):1762-1766, 2013

14. Quinlan JF, Duke D, Eustace S: Bertolotti's syndrome. A cause of back pain in young people. J Bone Joint Surg Br 88(9):11831186, 2006

15. Mitra R, Carlisle M: Bertolotti's syndrome: a case report. Pain Practice 9(2):152-154, 2009

16. Shibayama M, Ito F, Miura Y, Nakamura S, Ikeda S, Fujiwara K: Unsuspected reason for sciatica in Bertolotti's syndrome. J Bone Joint Surg Br 93(5):705-707, 2011

17. Dai L: Lumbosacral transitional vertebrae and low back pain. Bulletin (Hospital for Joint Diseases (New York, NY)) 58(4): 191-193, 1999

18. Hashimoto M, Watanabe O, Hirano H: Extraforaminal stenosis in the lumbosacral spine: efficacy of MR imaging in the coronal plane. Acta Radiol 37(5):610-613, 1996

19. Matsumoto M, Chiba K, Nojiri K, Ishikawa M, Toyama Y, Nishikawa Y:Extraforaminal entrapment of the fifth lumbar spinal nerve by osteophytes of the lumbosacral spine: anatomic study and a report of four cases. Spine (Phila Pa 1976) 27(6):169-173, 2002

20. Vergauwen S, Parizel PM, van Breusegem L, Van Goethem JW, Nackaerts Y, Van den Hauwe L, et al: Distribution and incidence of degenerative spine changes in patients with a lumbo-sacral transitional vertebra. Eur Spine J 6(3):168-172, 1997

21. Elster AD: Bertolotti's syndrome revisited. Transitional vertebrae of the lumbar spine. Spine (Phila Pa 1976) 14(12):1373-1377, 1989

22. Almeida DB, Mattei TA, Sória MG, Prandini MN, Leal AG, Milano JB, et al: Transitional lumbosacral vertebrae and low back pain: diagnostic pitfalls and management of Bertolotti's syndrome. Arq Neuropsiquiatr 67(2A):268-272, 2009

23. Santavirta S, Tallroth K, Ylinen P, Suoranta H: Surgical treatment of Bertolotti's syndrome. Follow-up of 16 patients. Arch Orthop Trauma Surg 112(2):82-87, 1993

24. Miyoshi Y, Yasuhara T, Date I: Posterior decompression of farout foraminal stenosis caused by a lumbosacral transitional vertebra. Neurol Med Chir (Tokyo) 51(2):153-156, 2011

25. Lee S, Kang JH, Srikantha U, Jang IT, Oh SH: Extraforaminal compression of the L-5 nerve root at the lumbosacral junction: clinical analysis, decompression technique, and outcome. J Neurosurg Spine 20(4):371-379, 2014

26. Yamada H, Yoshida M, Hashizume H, Minamide A, Nakagawa $\mathrm{Y}$, Kawai M, et al: Efficacy of novel minimally invasive surgery using spinal microendoscope for treating extraforaminal stenosis at the lumbosacral junction. J Spinal Disord Tech 25(5):268-276, 2012

27. Paudel B, Kim HS, Jang JS, Choi JH, Chung SK, Lee JS, et al: Percutaneous full endoscopic treatment of bertolotti syndrome: A report of three cases with technical note. J Neurol Surg A Cent Eur Neurosurg 78(6):566-571, 2017

28. Ju CI, Kim SW, Kim JG, Lee SM, Shin H, Lee HY: Decompressive L5 transverse processectomy for bertolotti's syndrome: A preliminary study. Pain Physician 20(6):923-932, 2017 